\title{
Analysis on Strong Tracking Filtering for Linear Dynamic Systems
}

\author{
Quanbo Ge, ${ }^{1}$ Teng Shao, ${ }^{1}$ Chenglin Wen, ${ }^{2}$ and Ruoyu Sun ${ }^{3}$ \\ ${ }^{1}$ Institute of Systems Science and Control Engineering, School of Automation, Hangzhou Dianzi University, Hangzhou 310018, China \\ ${ }^{2}$ College of Electrical Engineering, Henan University of Technology, Zhengzhou 450001, China \\ ${ }^{3}$ Department of Electrical and Computer Engineering, University of Minnesota, Twin Cities Campus, Minneapolis, MN 55414, USA
}

Correspondence should be addressed to Chenglin Wen; wencl@hdu.edu.cn

Received 28 February 2015; Accepted 6 August 2015

Academic Editor: David Bigaud

Copyright (C) 2015 Quanbo Ge et al. This is an open access article distributed under the Creative Commons Attribution License, which permits unrestricted use, distribution, and reproduction in any medium, provided the original work is properly cited.

\begin{abstract}
Strong tracking filtering (STF) is a popular adaptive estimation method to effectively deal with state estimation for linear and nonlinear dynamic systems with inaccurate models or sudden change of state. The key of the STF is to use a time-variant fading factor, which can be evaluated based on the current measurement innovation in real time, to forcefully correct one step state prediction error covariance. The strong tracking filtering technology has been extensively applied in many practical systems, but the theoretical analysis is highly lacking. In an effort to better understand STF, a novel analysis framework is developed for the strong tracking filtering and some new problems are discussed for the first time. For this, we propose a new perspective that correcting the state prediction error covariance by using the fading factor can be thought of directly modifying the state model by correcting the covariance of the process noise. Based on this proposed point of view, the conditions for the STF function to be effective are deeply analyzed in a certain linear dynamic system. Meanwhile, issues of false alarm and alarm failure are also briefly discussed for the strong tracking filtering function. Some numerical simulation examples are demonstrated to validate the results.
\end{abstract}

\section{Introduction}

State estimation is an important topic in many fields such as signal processing, target tracking, information fusion, and fault diagnosis [1-3]. The Kalman filter (KF) presented by Kalman (1960) is a strong and popular tool to estimate system state for linear systems and it is optimal in the sense of linear minimum mean square error (LMMSE) when the parameters of estimation models are accurate $[4,5]$. However, for most of the applications, it is difficult to obtain accurate system parameters, leading to mismatch of the practical system. It is challenging to study state estimation and fusion based on the Kalman filtering with inaccurate models.

Aiming at this problem mentioned above, a strong tracking filter (STF) was firstly presented in fault diagnosis field to deal with nonlinear state estimation with inaccurate models and the original purpose is only to overcome the shortcomings existing in the extended Kalman filter (EKF) for nonlinear systems [6-9]. For the EKF, in order to satisfy the running requirement of the linear Kalman filtering frame, the Taylor expansion technology is used to linearize the nonlinear systems. As a result of the linearization, the models used in the EKF algorithm mismatch the practical ones, which deteriorates the estimation and convergence performance of the EKF. To resolve this issue, the strong tracking filter, which is one of the adaptive filters, uses a time-variant fading factor based on the current measurement innovation to adjust dynamically the prediction error covariance matrix. As a result, the STF can obtain better estimation performance than the EKF when the used models mismatch the practical ones. Moreover, when the system state changes suddenly or target maneuver appears, the strong tracking filter can effectively track this change but the EKF or the linear KF cannot do. This is the reason why we say that the STF has strong tracking ability. It should be noted that the STF was presented for nonlinear systems, but its nature is to effectively deal with inaccuracy of the used models caused by linearization of nonlinear models. In other words, 
the motivation of the strong tracking filtering only focuses on the mismatching of models and is unrelated to linearity or nonlinearity of systems.

The strong tracking filtering has attracted considerable amount of attention from researchers and engineers in various fields, such as fault detection and diagnosis, target tracking, integrated navigation, observer design, and networked control, and many associated methods have been presented based on the STF [10-14]. It has been extensively used to design various state estimators and fusion estimators by combing other nonlinear filters $[15,16]$. Unfortunately, the effectiveness of the strong tracking filtering is usually validated by only simulations and experiments and theoretical analysis of the STF is very lacking. For the study on the STF theory, there are two main challenging issues:

(1) Rational formulation or explanation is always lacking on the strong tracking filtering technology. As a matter of fact, the time-variant strong tracking fading factor is subjectively introduced to modify computational formula of the prediction error covariance for the nature of the strong tracking filtering. All of succedent derivations on computational formula of the fading factor are based on this correction idea. Unfortunately, this subjective operation is short of support of some full explanations, such as how to explain the rationality of this fading factor and perfect its formulation and how to analyze the influence of the system models by this additive fading factor. Therefore, it is vital to perfect the strong tracking filtering theory.

(2) It is not clear whether the STF is effective in dealing with inaccurate parameters or models in all cases. Many studies with simulations and experiments have been presented to show that the strong tracking filtering is evidently effective for some cases with inaccurate system parameters or the sudden change of the system state. However, we are still not sure whether the STF can be active for all inaccurate cases. In other words, there is no theoretical evidence to completely guarantee that the strong tracking filtering can cover or deal with the inaccurate models in all cases. It is necessary to discuss condition under which the strong tracking filtering function is activated and analyze range of its effectiveness. Obviously, it is an interesting and innovative issue to perfect the strong tracking filtering theory.

To resolve these issues mentioned above, we provide a theoretical analysis of the STF for a kind of linear dynamic system and present a new point of view. It should be noted that the linear dynamic system is considered for simplicity in this paper. In this view, the effect of the strong tracking fading factor is equivalently thought to modify the state model (or the covariance of process noise) in real time based on the current measurement innovation. The analysis process and some discussions on the condition of the active strong tracking function are presented to explain our view and some numerical simulation examples are demonstrated to validate the results presented in this paper.

\section{System Models}

The linear estimation system models considered in this paper are described as follows:

$$
\begin{aligned}
& \mathbf{x}_{k}=\Phi_{k, k-1}^{a} \mathbf{x}_{k-1}+\mathbf{w}_{k, k-1} \\
& \mathbf{z}_{k}=\mathbf{H}_{k}^{a} \mathbf{x}_{k}+\mathbf{v}_{k}
\end{aligned}
$$

where (1) is called the state model and (2) is the measurement model. $\mathbf{x}_{k} \in \mathfrak{R}^{n \times 1}$ is a system state vector and $\Phi_{k, k-1}^{a} \epsilon$ $\mathfrak{R}^{n \times n}$ is the corresponding transition matrix of the system state from $k-1$ to $k . \mathbf{z}_{k} \in \mathfrak{R}^{p \times 1}$ is a measurement vector related to $\mathbf{x}_{k}$ and $\mathbf{H}_{k}^{a} \in \mathfrak{R}^{p \times n}$ is the associated measurement matrix. $\mathbf{w}_{k, k-1} \in \mathfrak{R}^{n \times 1}$, which is called process noise, is a Gaussian white noise with zero mean and covariance $\mathbf{Q}_{k, k-1}^{a}$. The measurement noise $\mathbf{v}_{k} \in \mathfrak{R}^{p \times 1}$ is a Gaussian vector with zero mean and covariance $\mathbf{R}_{k}^{a}$. Here, the superscript " $a$ " indicates accurate parameter vectors, that is, completely match practical systems. It is also assumed that initial state $\mathbf{x}_{0}$, whose mean and covariance are $\widehat{\mathbf{x}}_{0 \mid 0}$ and $\mathbf{P}_{0 \mid 0}$, respectively, is independent of $\mathbf{w}_{k, k-1}$ and $\mathbf{v}_{k}$ which are also independent of each other.

For practical applications, the mismatch is generally between ideal values $\Phi_{k, k-1}^{a}, \mathbf{H}_{k}^{a}, \mathbf{Q}_{k, k-1}^{a}$, and $\mathbf{R}_{k}^{a}$ and actual values of parameter vectors. In order to make a distinction between ideal and actual parameters, the following variables, $\Phi_{k, k-1}, \mathbf{H}_{k}, \mathbf{Q}_{k, k-1}$, and $\mathbf{R}_{k}$, are used to indicate the parameters in actual filtering process, which correspond to $\Phi_{k, k-1}^{a}, \mathbf{H}_{k}^{a}$, $\mathbf{Q}_{k, k-1}^{a}$, and $\mathbf{R}_{k}^{a}$, respectively. Note that the actual parameters are generally inaccurate due to many limitation factors for practical systems.

By replacing the ideal values in (1) and (2) by the actual values, we obtain the following actual estimation system:

$$
\begin{aligned}
& \mathbf{x}_{k}=\Phi_{k, k-1} \mathbf{x}_{k-1}+\mathbf{w}_{k, k-1}, \\
& \mathbf{z}_{k}=\mathbf{H}_{k} \mathbf{x}_{k}+\mathbf{v}_{k}
\end{aligned}
$$

where

$$
\begin{aligned}
E\left\{\mathbf{w}_{k, k-1}\right\} & =\mathbf{0}, \\
E\left\{\mathbf{w}_{k, k-1} \mathbf{w}_{k, k-1}^{T}\right\} & =\mathbf{Q}_{k, k-1}, \\
E\left\{\mathbf{v}_{k}\right\} & =\mathbf{0}, \\
E\left\{\mathbf{v}_{k} \mathbf{v}_{k}^{T}\right\} & =\mathbf{R}_{k} .
\end{aligned}
$$

As stated in the introduction, although the strong tracking filtering method was proposed for nonlinear systems, its nature is to deal with the inaccurate models induced by linearization operation with Taylor expansion. In other words, the STF can be used to deal with inaccuracy of the models no matter whether the system is linear or nonlinear. For the sake of convenience, we only consider the linear dynamic system shown by (3) in this paper and our results can be easily extended to nonlinear systems. 


\section{Strong Tracking Filter}

Initially, the strong tracking filtering was proposed in fault diagnosis field to overcome an issue of the nonlinear extended Kalman filter that the used linearization model in the EKF mismatches the practical system because higher order terms of Taylor expansion expression are neglected. As a result of this issue, the actually used parameters in the real-time filter are inaccurate in general. Consequently, the estimation performance of the EKF such as the estimation accuracy, convergence, and stability is poor. To resolve this issue, the STF was proposed to deal with the inaccuracy of the models and has been shown to be able to improve the estimation performance through simulations and experiments.

The idea of the STF is to compute a time-variant fading factor $\lambda_{k}$ in a heuristic way to adjust one step state prediction error covariance matrix in real time. For the linear dynamic system given by (3), the corrected state prediction error covariance matrix is $[6,7,15,16]$

$$
\mathbf{P}_{k \mid k-1}^{s}=\lambda_{k} \Phi_{k, k-1} \mathbf{P}_{k-1 \mid k-1} \Phi_{k, k-1}^{T}+\mathbf{Q}_{k, k-1},
$$

where $\lambda_{k}$ is computed by solving the following optimization problem:

$$
\begin{aligned}
\text { Minimize } & E\left\{\left[\mathbf{x}_{k}-\widehat{\mathbf{x}}_{k \mid k}\right]\left[\mathbf{x}_{k}-\widehat{\mathbf{x}}_{k \mid k}\right]^{T}\right\} \\
\text { s.t. } & E\left\{v_{k+m} v_{k}^{T}\right\}=\mathbf{0}, \quad k=1,2, \ldots ; m \geq 1,
\end{aligned}
$$

where the measurement innovation $v_{k}=\mathbf{z}_{k}-\widehat{\mathbf{z}}_{k \mid k-1}$ and $\widehat{\mathbf{z}}_{k \mid k-1}$ is the measurement prediction. The cost function in (6) is the optimal rule of state estimation, and the constraint means that the innovations at different times should be orthogonal in order to achieve the optimality of the linear minimum mean square error $[2,6]$. This constraint helps us extract available information from real-time measurements as much as possible. If they are not orthogonal, the available information contained in the measurement are not fully used for filtering estimation.

While solving the optimization problem (6) exactly is challenging, a popular choice of the suboptimal $\lambda_{k}$ is $[2,6]$

$$
\lambda_{k}= \begin{cases}c_{k}, & c_{k}>1, \\ 1, & c_{k} \leq 1,\end{cases}
$$

where

$$
c_{k}=\frac{\operatorname{Tr}\left(\mathbf{N}_{k}\right)}{\operatorname{Tr}\left(\mathbf{M}_{k}\right)},
$$

in which "Tr" is the trace operation and

$$
\begin{gathered}
\mathbf{N}_{k}=\mathbf{V}_{0, k}-\beta^{*} \mathbf{R}_{k}-\mathbf{H}_{k} \mathbf{Q}_{k, k-1} \mathbf{H}_{k}^{T}, \\
\mathbf{M}_{k}=\mathbf{H}_{k} \Phi_{k, k-1} \mathbf{P}_{k-1 \mid k-1} \Phi_{k, k-1}^{T} \mathbf{H}_{k}^{T}, \\
\mathbf{V}_{0, k}= \begin{cases}v_{1} v_{1}^{T}, & k=1, \\
\frac{\left(\rho \mathbf{V}_{0, k-1}+v_{k} v_{k}^{T}\right)}{(1+\rho)}, & k>1,\end{cases}
\end{gathered}
$$

where $0<\rho \leq 1$ and $\beta^{*} \geq 1$.
In most current studies, the STF with the fading factor shown by (7) has been extensively applied in linear and nonlinear system, and simulations results obviously show its effectiveness [6-9]. Unfortunately, the theoretical analysis of the rationality of the STF idea is still missing. More specifically, as the purpose of the strong tracking filtering is to deal with the mismatched models cases, it is exceedingly necessary to make clear how the subjective operation of the strong tracking fading factor reduces the influences of the inaccuracy and improves the system performance. In addition, it is also not known whether this fading factor is sensitive for all of cases with the mismatched models up to now, which necessitates a discussion on the conditions of effectiveness of the strong tracking function. Motivated by these questions, we present a novel point of view on the principle of the strong tracking filtering. Some related issues are also discussed in the subsequent sections which help us better understand the strong tracking filtering theory.

\section{A View of the Strong Tracking Filtering}

In order to provide a rational explanation on the effect of the strong tracking fading factor, a novel view on the STF is presented in this section. The essence of this view is that modifying the prediction error covariance is effectively to dynamically correct and compensates the state model. It is helpful to understand the rationality and the nature of the strong tracking filtering theory from this novel view. Certainly, it also contributes to completeness of the strong tracking filtering theory.

The computation in (5) of the corrected prediction error covariance matrix can be rewritten as

$$
\mathbf{P}_{k \mid k-1}^{s}=\Phi_{k, k-1} \mathbf{P}_{k-1 \mid k-1} \Phi_{k, k-1}^{T}+\mathbf{Q}_{k, k-1}^{s},
$$

where

$$
\begin{aligned}
& \mathbf{Q}_{k, k-1}^{s}=\mathbf{Q}_{k, k-1}+\mathbf{Q}_{k, k-1}^{\lambda}, \\
& \mathbf{Q}_{k, k-1}^{\lambda}=\left(\lambda_{k}-1\right) \Phi_{k, k-1} \mathbf{P}_{k-1 \mid k-1} \Phi_{k, k-1}^{T} .
\end{aligned}
$$

According to the basic principle of linear minimum mean square error (LMMSE) estimation, the corrected prediction error covariance matrix can be directly derived by the following formula:

$$
\begin{aligned}
\mathbf{P}_{k \mid k-1}^{s} & =E\left\{\left[\mathbf{x}_{k}-\widehat{\mathbf{x}}_{k \mid k-1}\right]\left[\mathbf{x}_{k}-\widehat{\mathbf{x}}_{k \mid k-1}\right]^{T}\right\} \\
& =\Phi_{k, k-1} \mathbf{P}_{k-1 \mid k-1} \Phi_{k, k-1}^{T}+E\left\{\mathbf{w}_{k, k-1} \mathbf{w}_{k, k-1}^{T}\right\} .
\end{aligned}
$$

Comparing (11) and (13), we get

$$
E\left\{\mathbf{w}_{k, k-1} \mathbf{w}_{k, k-1}^{T}\right\}=\mathbf{Q}_{k, k-1}^{s} .
$$

Note that the covariance of the process noise $\mathbf{w}_{k, k-1}$ is $\mathbf{Q}_{k, k-1}$ in the initial state model. Therefore, (14) can be interpreted as that the covariance of the process noise in the state model is corrected by using the dynamic fading factor, which is based on the real-time measurement innovation. As a result, the state model is naturally improved by correcting 
the covariance of the process noise. In other words, our view is that the nature of the correction operation of the prediction error covariance is to dynamically modify the state model for the strong tracking filtering. Its purpose is to make the modified state model close to the practical one as soon as possible. Clearly, the modification of the state model depends closely on the fading factor and the real-time filtering process for this view. The presented view helps to explain the phenomenon that the STF is particularly effective for inaccurate state models and sudden change of the state $[7,13,15]$. In fact, the state model is improved in the STF by correcting the computation of the prediction error covariance. In order to further make clear the principle of the strong tracking filtering, we discuss the condition under which the function of the strong tracking filtering is active in the next section based on the presented view in this section.

\section{Analysis on Efficiency Condition of the Strong Tracking Filtering}

In this section, we only focus on the analysis on active condition of the strong tracking function under the new view given in Section 4. In terms of principle of the STF, the strong tracking function is active when the fading factor $\lambda_{k}>1$. To directly see what the STF factor expression means, we rewrite $\lambda_{k} \geq 1$ as

$$
\frac{\operatorname{Tr}\left(\mathbf{N}_{k}\right)}{\operatorname{Tr}\left(\mathbf{M}_{k}\right)}>1
$$

which is equivalent to

$$
\operatorname{Tr}\left(\mathbf{N}_{k}\right)-\operatorname{Tr}\left(\mathbf{M}_{k}\right)>0
$$

Using (9), we further obtain

$$
\begin{aligned}
\operatorname{Tr} & \left.\mathbf{N}_{k}\right)-\operatorname{Tr}\left(\mathbf{M}_{k}\right) \\
= & \operatorname{Tr}\left(\mathbf{V}_{0, k}-\beta^{*} \mathbf{R}_{k}-\mathbf{H}_{k} \mathbf{Q}_{k, k-1} \mathbf{H}_{k}^{T}\right) \\
& \quad-\operatorname{Tr}\left(\mathbf{H}_{k} \Phi_{k, k-1} \mathbf{P}_{k-1 \mid k-1} \Phi_{k, k-1}^{T} \mathbf{H}_{k}^{T}\right)>0 .
\end{aligned}
$$

Equation (17) provides a criterion to determine whether the strong tracking function should be active. It also shows that the effective strong tracking fading factor depends closely on the four parameter vectors $\Phi_{k, k-1}, \mathbf{H}_{k}, \mathbf{Q}_{k, k-1}$, and $\mathbf{R}_{k}$ in practical estimation systems.

In the rest of this section, we present an analysis based on the proposed point of view to solely correct the covariance of the process noise in scalar and vector cases, respectively.

5.1. Scalar Case. If the system parameters are all scalar, namely, $n=p=1$, (17) can be rewritten as

$$
\begin{aligned}
\operatorname{Tr}\left(\mathbf{N}_{k}\right)-\operatorname{Tr}\left(\mathbf{M}_{k}\right)= & \mathbf{N}_{k}-\mathbf{M}_{k} \\
= & \mathbf{V}_{0, k}-\beta^{*} \mathbf{R}_{k}-\mathbf{H}_{k}^{2} \mathbf{Q}_{k, k-1} \\
& -\mathbf{H}_{k}^{2} \Phi_{k, k-1}^{2} \mathbf{P}_{k-1 \mid k-1}>0 .
\end{aligned}
$$

Because our view focuses only on the covariance of the process noise, we can obtain the following proposition on a judging condition that the strong tracking filtering becomes active from (18).

Proposition 1. When the strong tracking function is active, it must satisfy an inequality condition as follows:

$$
\mathbf{Q}_{k, k-1}<\mathbf{Q}_{k, k-1}^{u}
$$

where

$$
\mathbf{Q}_{k, k-1}^{u}=\mathbf{H}_{k}^{-2} \mathbf{V}_{0, k}-\beta^{*} \mathbf{H}_{k}^{-2} \mathbf{R}_{k}-\Phi_{k, k-1}^{2} \mathbf{P}_{k-1 \mid k-1} .
$$

This conclusion given in Proposition 1 can be proved directly from (18). Here, $\mathbf{Q}_{k, k-1}^{u}$, for which " $u$ " means upper bound, is a scalar threshold to decide whether the strong tracking function is efficient and the state model matches the practical system. If $\mathbf{Q}_{k, k-1}$ is less than $\mathbf{Q}_{k, k-1}^{u}$, the used models mismatch the practical system and the STF function should be active. On the contrary, it indicates that the used system models can fully formulate the uncertainty included in the practical system.

Next, we illustrate how to derive the expression of STF factor in (7) for the case $\lambda_{k}>1$ by letting the "process noise covariance" $\mathbf{Q}_{k, k-1}^{s}$ be equal to some specific value, as a heuristic replacement of minimizing $\mathbf{Q}_{k, k-1}^{s}$ in problem (6). The strong tracking filtering can successfully correct the state model by compensating and amplifying the covariance of the process noise from (11) and (12). From (19), it also shows that the corrected model in the STF matches the practical system when the modified covariance $\mathbf{Q}_{k, k-1}^{s}$ reaches $\mathbf{Q}_{k, k-1}^{u}$; that is,

$$
\mathbf{Q}_{k, k-1}^{s}=\mathbf{Q}_{k, k-1}^{u}
$$

In order to satisfy the correction requirement, this covariance of the process noise should be compensated and the increment $\mathbf{Q}_{k, k-1}^{s, c}$ can be computed by

$$
\mathbf{Q}_{k, k-1}^{s, c}=\mathbf{Q}_{k, k-1}^{u}-\mathbf{Q}_{k, k-1}>0 \text {. }
$$

Combining (11), (12), (21), and (22), we get

$$
\mathbf{Q}_{k, k-1}^{s, c}=\mathbf{Q}_{k, k-1}^{\lambda}>0 .
$$

Clearly, this result accords with the view in Section 4 and one clearly has that $\lambda_{k}>1$.

Plugging (21) into (12), we get

$$
\mathbf{Q}_{k, k-1}^{u}=\mathbf{Q}_{k, k-1}+\mathbf{Q}_{k, k-1}^{\lambda}
$$

Plugging (20) and the second equation of (12) into (24), we get

$$
\lambda_{k}=\frac{\mathbf{V}_{0, k}-\beta^{*} \mathbf{R}_{k}-\mathbf{H}_{k}^{2} \mathbf{Q}_{k, k-1}}{\Phi_{k, k-1}^{2} \mathbf{H}_{k}^{2} \mathbf{P}_{k-1 \mid \mathrm{k}-1}}>1
$$

The expression of $\lambda_{k}>1$ given in (25) is consistent with the result computed by (7)-(10) for the scalar case because Proposition 1 is derived from the computation formulas of 
the strong tracking fading factor. Meanwhile, it also shows that Proposition 1 is necessary and sufficient.

This proposition is more helpful to understand the principle of the STF than the original computation description equations (7)-(10). When $\mathbf{Q}_{k, k-1}<\mathbf{Q}_{k, k-1}^{u}$, the STF uses $\mathbf{Q}_{k, k-1}^{\lambda}$ based on the current measurement innovation to correct and compensate the original state model or the covariance of the process noise, which can better match the characteristics of the practical system at the current time.

5.2. Vector Case. Based on (17) and the analysis of the scalar case in Section 5.1, we also have a similar result for the vector case, which is shown by the following proposition.

Proposition 2. Similar to the scalar case, if the strong tracking function is effective for the vector case, it must satisfy the following inequality:

$$
\mathbf{f}\left(\mathbf{Q}_{k, k-1}\right)<\mathbf{f}\left(\mathbf{Q}_{k, k-1}^{u, v}\right)
$$

where

$$
\begin{aligned}
\mathbf{f}\left(\mathbf{Q}_{k, k-1}\right)= & \operatorname{Tr}\left(\mathbf{H}_{k} \mathbf{Q}_{k, k-1} \mathbf{H}_{k}^{T}\right), \\
\mathbf{f}\left(\mathbf{Q}_{k, k-1}^{u, v}\right)= & \operatorname{Tr}\left(\mathbf{V}_{0, k}-\beta^{*} \mathbf{R}_{k}\right) \\
& -\operatorname{Tr}\left(\mathbf{H}_{k} \Phi_{k, k-1} \mathbf{P}_{k-1 \mid k-1} \Phi_{k, k-1}^{T} \mathbf{H}_{k}^{T}\right),
\end{aligned}
$$

where $\mathbf{Q}_{k, k-1}^{u, v}$ is the upper bound for the vector case and " $v$ " indicates the vector case.

Similar to (21), we have

$$
\mathbf{f}\left(\mathbf{Q}_{k, k-1}^{s}\right)=\mathbf{f}\left(\mathbf{Q}_{k, k-1}^{u, v}\right),
$$

where $\mathbf{f}\left(\mathbf{Q}_{k, k-1}^{s}\right)=\operatorname{Tr}\left(\mathbf{H}_{k} \mathbf{Q}_{k, k-1}^{s} \mathbf{H}_{k}^{T}\right)$.

Also, we have

$$
\mathbf{f}\left(\mathbf{Q}_{k, k-1}^{s, c}\right)=\mathbf{f}\left(\mathbf{Q}_{k, k-1}^{u, v}\right)-\mathbf{f}\left(\mathbf{Q}_{k, k-1}\right)=\mathbf{f}\left(\mathbf{Q}_{k, k-1}^{\lambda}\right)>0 .
$$

Similar to (24), we have

$$
\mathbf{f}\left(\mathbf{Q}_{k, k-1}^{u, v}\right)=\mathbf{f}\left(\mathbf{Q}_{k, k-1}\right)+\mathbf{f}\left(\mathbf{Q}_{k, k-1}^{\lambda}\right) \text {. }
$$

Moreover, by combing (12) and (30), one obviously has $\lambda_{k}>$ 1. According to (12), (26), (27), and (31), we can obtain the computation formula of $\lambda_{k}$ as follows:

$$
\lambda_{k}=\frac{\operatorname{Tr}\left(\mathbf{V}_{0, k}-\beta^{*} \mathbf{R}_{k}-\mathbf{H}_{k} \mathbf{Q}_{k, k-1} \mathbf{H}_{k}^{T}\right)}{\operatorname{Tr}\left(\mathbf{H}_{k} \Phi_{k, k-1} \mathbf{P}_{k-1 \mid k-1} \Phi_{k, k-1}^{T} \mathbf{H}_{k}^{T}\right)}>1 .
$$

Clearly, $\lambda_{k}>1$ in (32) and this result is also consistent with the computation formulas of $\lambda_{k}$ given by (7)-(10). Likewise, the conclusion given by this proposition is also necessary and sufficient, which is similar to the scalar case.

\section{Discussion on Defect of the STF to Detect Model Mismatch}

The intuitive expression on the condition of active strong tracking function is studied in last section. Another interesting and important question is whether the STF can detect the model mismatch in all cases. This is the detectability of the STF to mismatched models which is discussed in this section.

The decision criteria of the strong tracking filtering, that is, (17), is equivalent to

$$
\begin{aligned}
& \operatorname{Tr}\left(\mathbf{V}_{0, k}-\beta^{*} \mathbf{R}_{k}-\mathbf{H}_{k} \mathbf{Q}_{k, k-1} \mathbf{H}_{k}^{T}\right) \\
& \quad>\operatorname{Tr}\left(\mathbf{H}_{k} \Phi_{k, k-1} \mathbf{P}_{k-1 \mid k-1} \Phi_{k, k-1}^{T} \mathbf{H}_{k}^{T}\right)
\end{aligned}
$$

The decision rule given by (33) may lead to a wrong result (false alarm), for which the strong tracking function should be also activated when both models are absolutely accurate. Obviously, it does not accord with the original motivation of the strong tracking filtering theory. As a result, there is a conflict that the linear Kalman filter is optimal and best linear unbiased when all of parameters absolutely match the practical system. The possibility of false alarm shows that the strong tracking filtering performance is far from desirable under some specified conditions.

If the strong tracking function is not valid, it must satisfy the following condition:

$$
\begin{aligned}
\operatorname{Tr} & \left(\mathbf{V}_{0, k}-\beta^{*} \mathbf{R}_{k}-\mathbf{H}_{k} \mathbf{Q}_{k, k-1} \mathbf{H}_{k}^{T}\right) \\
& <\operatorname{Tr}\left(\mathbf{H}_{k} \Phi_{k, k-1} \mathbf{P}_{k-1 \mid k-1} \Phi_{k, k-1}^{T} \mathbf{H}_{k}^{T}\right)
\end{aligned}
$$

Clearly, there are many cases on $\mathbf{Q}_{k, k-1}$ and $\mathbf{R}_{k}$ to satisfy inequality (34). Next, we give a simple analysis to show functional defect of the STF in dealing with inaccurate models.

Denote $\mathbf{Q}_{k, k-1}^{\circ}$ and $\mathbf{R}_{k}^{\circ}$ as other values of the covariances of the process noise and the measurement noise, respectively, and suppose

$$
\begin{aligned}
\mathbf{Q}_{k, k-1}^{\circ} & =\mathbf{Q}_{k, k-1}+\Delta \mathbf{Q}_{k, k-1}, \\
\mathbf{R}_{k}^{\circ} & =\mathbf{R}_{k}+\Delta \mathbf{R}_{k},
\end{aligned}
$$

where $\Delta \mathbf{Q}_{k, k-1}$ and $\Delta \mathbf{R}_{k}$ are differences which deviate from $\mathbf{Q}_{k, k-1}$ and $\mathbf{R}_{k}$, respectively. Then, by using (35) in (34), left expression $\mathbf{L}\left(\mathbf{Q}_{k, k-1}, \mathbf{R}_{k}\right)$ of (34) becomes

$$
\begin{gathered}
\mathbf{L}\left(\mathbf{Q}_{k, k-1}^{\circ}, \mathbf{R}_{k}^{\circ}\right)=\operatorname{Tr}\left(\mathbf{V}_{0, k}-\beta^{*}\left(\mathbf{R}_{k}+\Delta \mathbf{R}_{k}\right)\right. \\
\left.-\mathbf{H}_{k}\left(\mathbf{Q}_{k, k-1}+\Delta \mathbf{Q}_{k, k-1}\right) \mathbf{H}_{k}^{T}\right) .
\end{gathered}
$$

The system models should be considered to be accurate for the STF and the basic Kalman filter will be run as long as $\mathbf{L}\left(\mathbf{Q}_{k, k-1}^{\circ}, \mathbf{R}_{k}^{\circ}\right)=\mathbf{L}\left(\mathbf{Q}_{k, k-1}, \mathbf{R}_{k}\right)$, which, according to (34) and (36), is equivalent to

$$
\operatorname{Tr}\left(\beta^{*} \Delta \mathbf{R}_{k}+\mathbf{H}_{k} \Delta \mathbf{Q}_{k, k-1} \mathbf{H}_{k}^{T}\right)=0 .
$$

Obviously, there exist many possible pairs of $\left(\Delta \mathbf{Q}_{k, k-1}\right.$, $\Delta \mathbf{R}_{k}$ ) that satisfy (37). In other words, it is possible that the inaccuracies on the covariances of the process and measurement noises can offset each other. This reveals, for the first time, an interesting phenomenon that the current 
STF theory may lead to possible missing alarm (or alarm failure) to model inaccuracy. Meanwhile, it also shows that the current strong tracking filtering cannot detect inaccuracy of individual parameter since its decision criteria depends on a combination of all of model parameters. In summary, the STF cannot deal with the mismatched models in all cases because of possible false and missing alarms and thus the application range of the current STF is limited. Therefore, it is necessary to further improve the STF to be more intelligent so that it can deal with more cases of inaccurate models.

\section{Simulation Examples}

There are three simulation examples which are used to validate the analysis and conclusions presented in this paper. In this section, a target tracking scene is considered and the target state is $\mathbf{x}_{k}=\left[\mathbf{x}_{1, k} ; \mathbf{x}_{2, k}\right]$, where $\mathbf{x}_{1, k}$ is target displacement and $\mathbf{x}_{2, k}$ indicates its velocity. The constant velocity model is used and

$$
\begin{aligned}
\Phi_{k, k-1}^{a} & =\left[\begin{array}{ll}
1 & T \\
0 & 1
\end{array}\right], \\
\mathbf{Q}_{k, k-1}^{a} & =\left[\begin{array}{cc}
\frac{T^{3}}{3} & \frac{T^{2}}{2} \\
\frac{T^{2}}{2} & T
\end{array}\right],
\end{aligned}
$$

where $T=1 \mathrm{~s}$ is the sample period. The ideal parameters of measurement model are

$$
\begin{aligned}
\mathbf{H}_{k}^{a} & =\left[\begin{array}{ll}
1 & 0
\end{array}\right], \\
\mathbf{R}_{k}^{a} & =1 .
\end{aligned}
$$

The initial state estimate and its associated covariance are

$$
\begin{aligned}
\widehat{\mathbf{x}}_{0 \mid 0} & =\left[\begin{array}{c}
0 \\
0.1
\end{array}\right], \\
\mathbf{P}_{0 \mid 0} & =\left[\begin{array}{ll}
10 & 0 \\
0 & 1
\end{array}\right] .
\end{aligned}
$$

Example 1. In this example, we verify the functional equivalence of the two correction ways, which are the current STF to directly modify the prediction error covariance matrix (STF1) and the way to correct the covariance of the process noise (STF2), respectively. Two different cases are considered such as inaccurate covariances of process and measurement noises (Case 1) and the sudden change of state (Case 2) in this simulation and all results are obtained by averaging over 100 Monte-Carlo simulations runs.

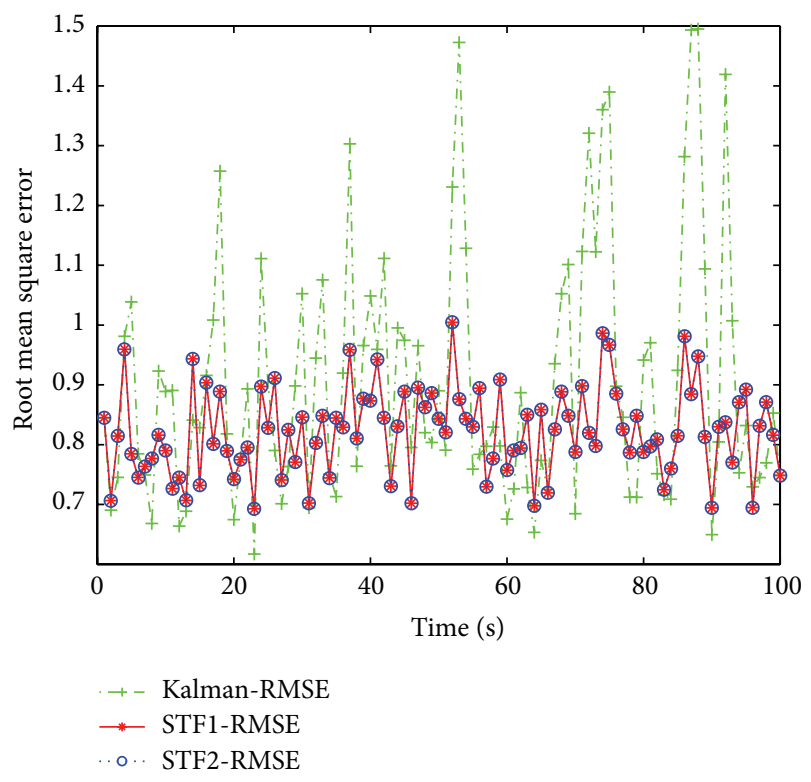

FIGURE 1: Root mean square errors of $\mathbf{x}_{1, k}$.

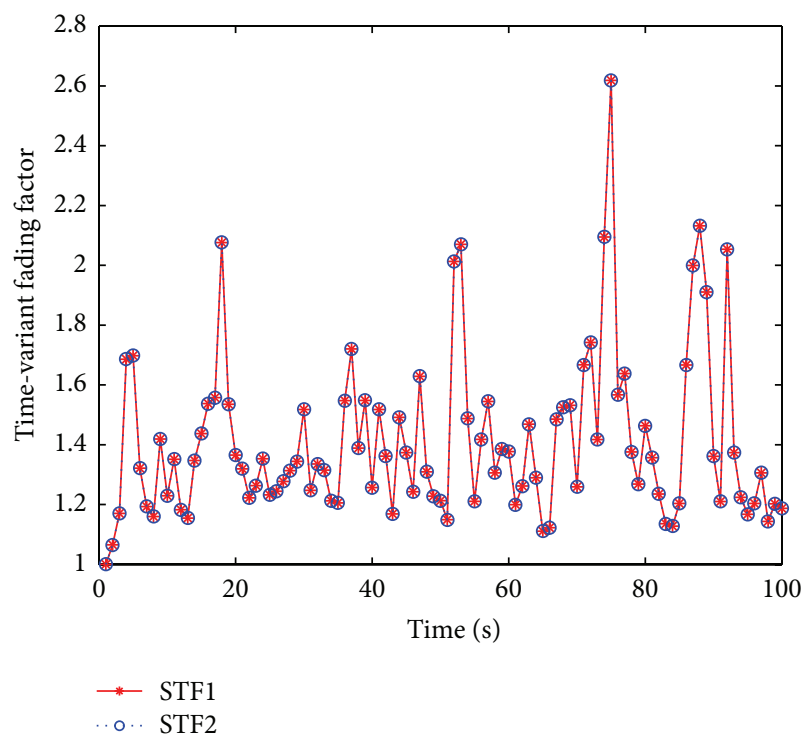

FIGURE 2: Time-variant fading factors.

Case 1. In this case, the inaccurate values on the covariances of process and measurement noises are used in the practical filtering process, which are

$$
\begin{aligned}
\mathbf{Q}_{k, k-1} & =\left[\begin{array}{cc}
\frac{T^{3}}{4} & T^{2} \\
T^{2} & \frac{T}{2}
\end{array}\right], \\
\mathbf{R}_{k} & =0.8 .
\end{aligned}
$$

For the strong tracking filtering, $\beta^{*}=1.2$ and $\rho=0.95$. The simulation results are plotted in Figures $1-3$. From Figure 1, we know that when the parameters are mismatched 


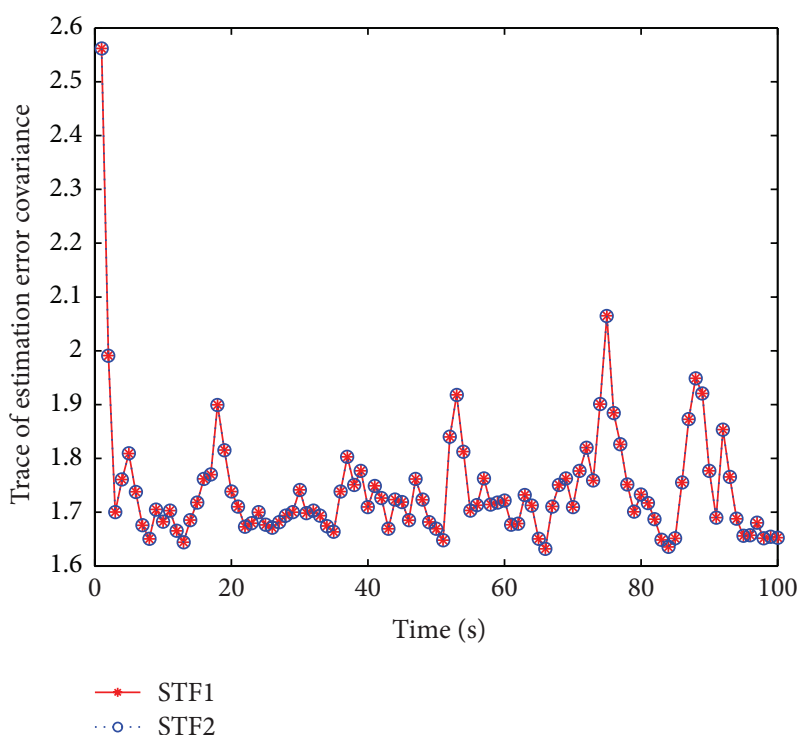

FIgURE 3: Traces of estimation error covariances.

or inaccurate in the practical filtering process, the estimation accuracy of the standard Kalman filter is worse than the strong tracking filters with time-variant fading factor. It shows that the strong tracking filter has the adaptive adjustment ability to deal with inaccurate models. Figures 2 and 3 indicate that the fading factors of two strong tracking filters are the same and the estimation accuracies of two strong tracking filters are equivalent, respectively. Clearly, the simulation results are consistent with the theoretical analysis in this paper.

Case 2. Different from Case 1, we add the sudden change of state in the state model as in $[40,60]$. A constant amplitude of the sudden change of state is $[6 ; 5]$ from time 40 to time 50 and $[30 ; 5]$ at other times. The simulation results are shown by Figures $4-7$.

Clearly, Figure 4 shows that the accuracies of two strong tracking filters are better than that of the standard Kalman filter when the sudden change of the state appears. The Kalman filter cannot track the sudden change of the state but the two tracking filters can. In other words, the strong tracking filtering can also deal with this kind of model mismatching but the standard Kalman filter cannot. From Figures 5 and 6 , the estimation results are equivalent for two STFs. Similar to Case 1, the fading factors are the same from Figure 7.

Example 2. In this example, the functional equivalence between (7)-(10) and (17) is validated. The inaccuracy of the covariance of measurement noise is only considered and $\mathbf{Q}_{k, k-1}$ refers to Case 1 in Example 1. The values of $\lambda_{k}$ in (7) and $\mathrm{TR}_{k}=\operatorname{Tr}\left(\mathbf{N}_{k}\right)-\operatorname{Tr}\left(\mathbf{M}_{k}\right)$ in (17) are both computed in the procedure. Simulation result is shown by Figure 8. In this figure, the blue full line indicates 1 and the value of red dotted line is 0 . Then, we have the following conclusion; namely, if $\lambda_{k} \geq 1$, then $\mathrm{TR}_{k} \geq 0$ and if $\lambda_{k}=1$, then $\mathrm{TR}_{k} \leq 0$. This is because (20) is equivalently derived from the computation

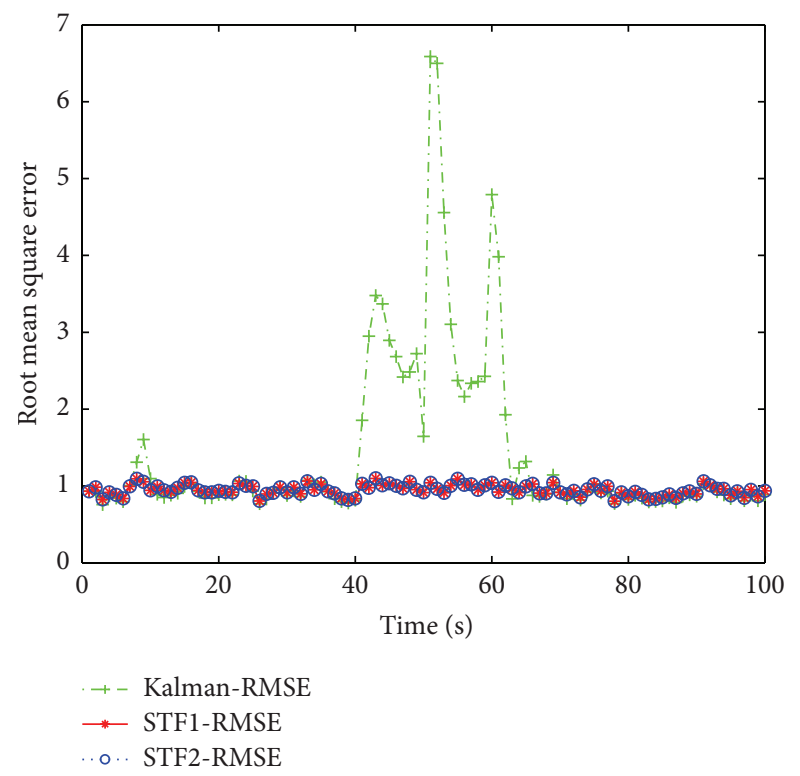

FIgURE 4: Root mean square errors of $\mathbf{x}_{1, k}$.

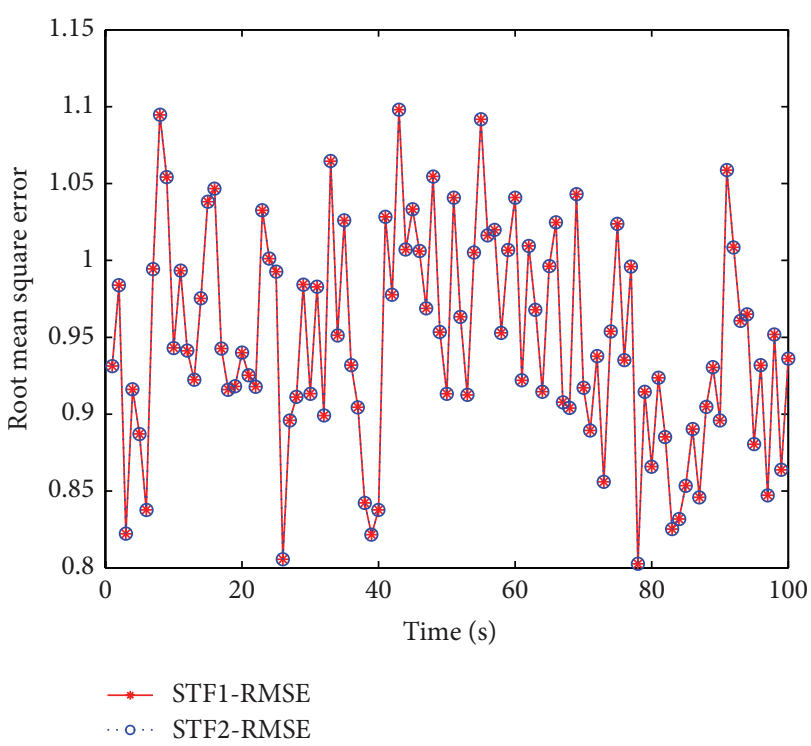

FIGURE 5: Root mean square errors of $\mathbf{x}_{1, k}$.

formulas of the time-variant fading factor. Obviously, this simulation result is also consistent with the corresponding theoretical analysis given in this paper.

Example 3. This example is demonstrated to validate the false alarm and the alarm failure (or the missing alarm) problem discovered in Section 6. Certainly, the condition expressed by (34) should be guaranteed. The simulation results are given by Figures 9, 10, and 11 and Table 1 . Figure 9 and Table 1 show that the strong tracking fading factor is possibly greater than one when all parameters are accurate; namely, the false alarm exists for the current strong tracking filtering. The red dotted line shown in Figure 10 is to express (37) with variables $\Delta \mathbf{Q}_{k, k-1}$ and $\Delta \mathbf{R}_{k}$. The result shown in Figure 11 indicates 


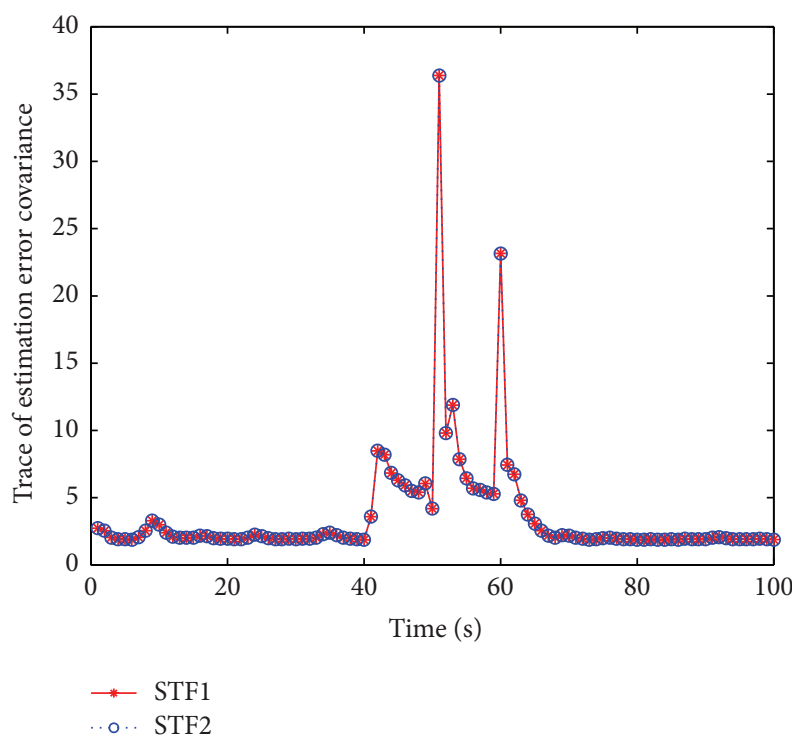

FIgURE 6: Traces of estimation error covariances.

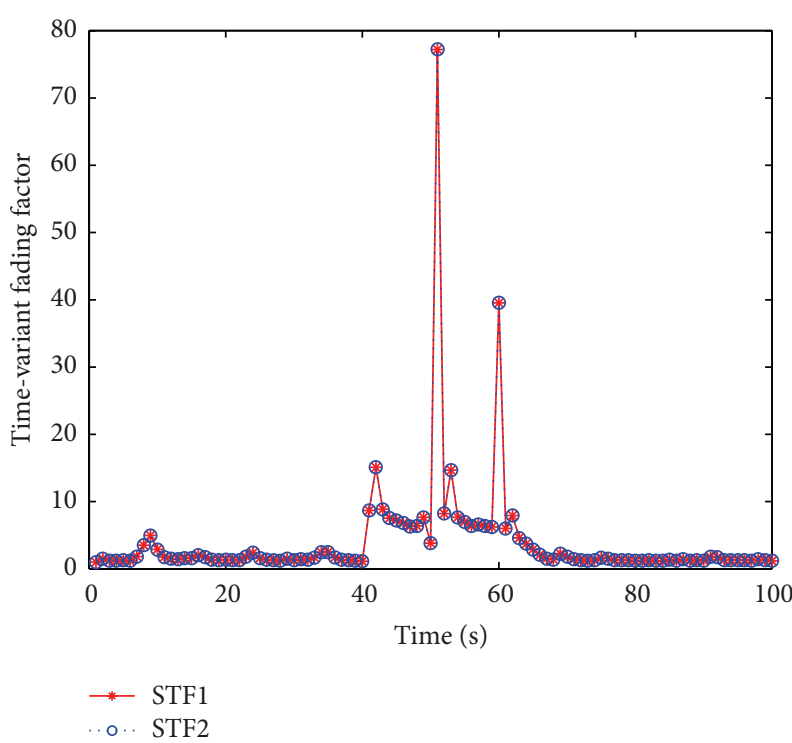

FIGURE 7: Time-variant fading factors.

TABLE 1: Fading factor values which are greater than one.

\begin{tabular}{lcccccc}
\hline Time & 37 & 38 & 39 & 58 & 59 & 60 \\
\hline $\mathbf{N}_{k}$ & 4.0465 & 4.6505 & 3.1386 & 4.6512 & 6.8338 & 4.5737 \\
$\mathbf{M}_{k}$ & 2.7775 & 2.9535 & 3.0565 & 2.7775 & 3.0243 & 3.3114 \\
$\lambda_{k}$ & 1.4569 & 1.5746 & 1.0269 & 1.6746 & 2.2596 & 1.3812 \\
\hline
\end{tabular}

that when the constraint given by (37) is satisfied, the strong tracking function is disabled for the mismatched system models; namely, $\lambda=1$. It also shows that the alarm failure possibly appears for the conventional strong tracking filtering theory. Accordingly, the analytic result given in Section 6 is validated.

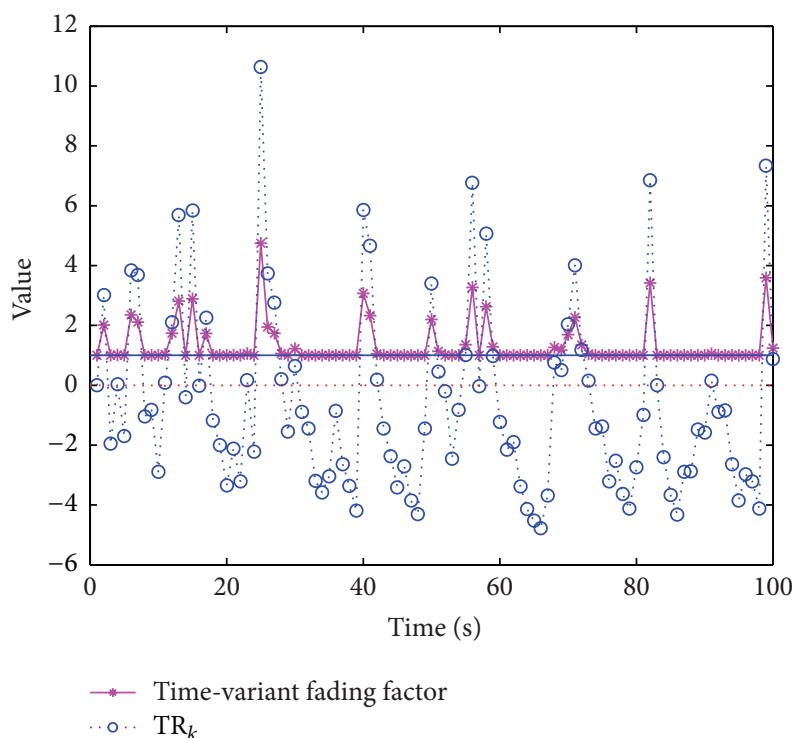

FIGURE 8: Results of fading factor and $\mathrm{TR}_{k}$.

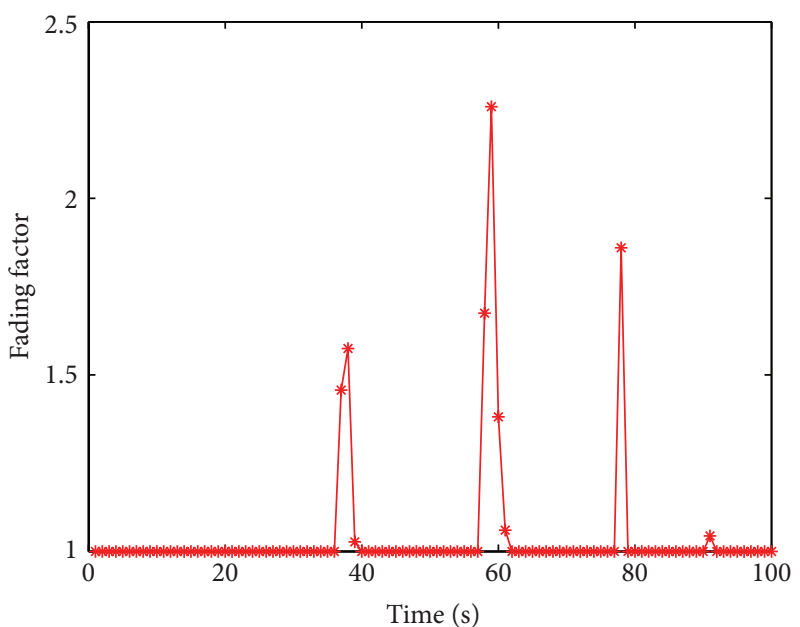

FIGURE 9: Fading factors of the STF with accurate models.

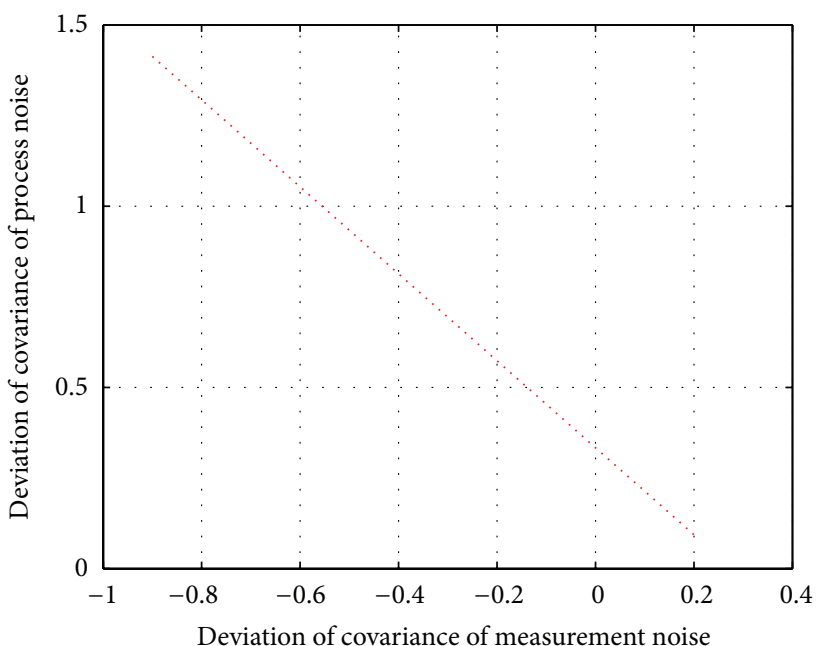

Figure 10: Curve with two parameter vectors which satisfy (37). 


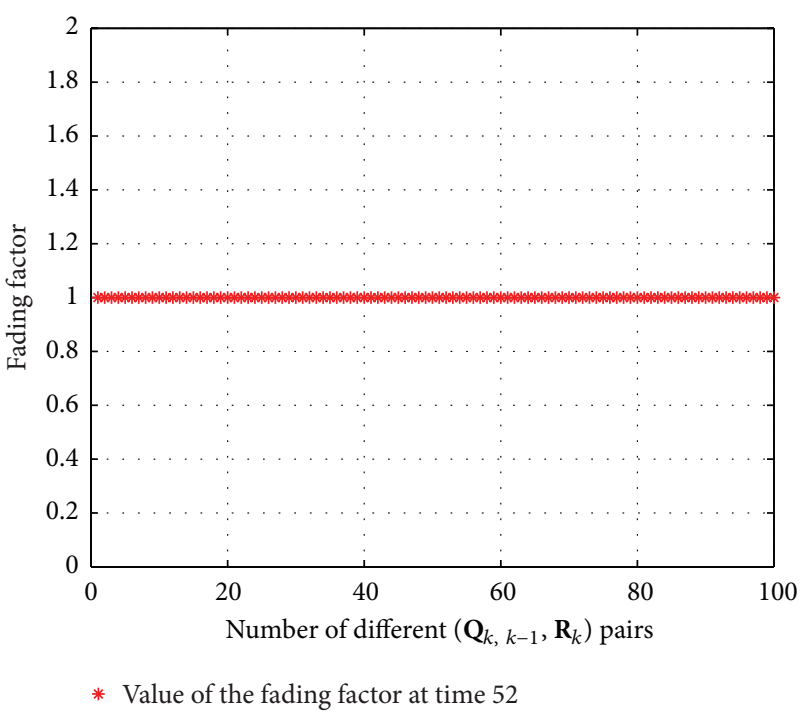

FIGURE 11: Fading factor values satisfy condition equation (37).

\section{Conclusions}

Aiming at a kind of linear dynamic system, we present a new and interesting view on the strong tracking filtering theory which is quite effective for some state estimation systems with the inaccurate parameters and the sudden change of state. From this point of view, correcting the state prediction error covariance by using the fading factor in the traditional strong tracking filter is considered to improve the process noise covariance of the state model. A more direct sufficient condition to activate the strong tracking function (the fading factor is greater than one) is presented and a detailed analysis with scalar and vector cases are also provided. The analysis results show that the conventional tracking filtering method is not always available because it must satisfy a certain condition which cannot cover all of the situations. Additionally, the conventional strong tracking filter cannot detect which parameter is inaccurate when the strong tracking function is active. We expect that our studies can provide new insight into the STF theory. In the future, it is interesting and challenging to further develop the analysis on the principle of the strong tracking filtering theory from different angles of view and further improve the strong tracking filtering theory.

\section{Conflict of Interests}

The authors declare that there is no conflict of interests regarding the publication of this paper.

\section{Acknowledgments}

The authors would like to acknowledge Dr. Z. S. Duan of Xi'an Jiaotong University in China. This work was partially supported by National Nature Science Fund of China (NSFC) (Grant nos. 61172133, 61273075, 61333011, and 61333005) and
Master Fund of Hangzhou Dianzi University (Grant no. yxlw2015014).

\section{References}

[1] Y. He, G. H. Wang, and X. Guan, Information Fusion Theory with Applications, Publishing House of Electronics Industry, Beijing, China, 2010.

[2] D. H. Zhou and Y. Y. Zhong, Modern Fault Diagnosis and Tolerancefault Control, Publishing House of Tsinghua University, Beijing, China, 2000.

[3] Y. Bar-Shalom, X. R. Li, and T. Kirubarajan, Estimation with Applications to Tracking and Navigation: Theory Algorithms and Software, John Wiley \& Sons, 2004.

[4] R. E. Kalman, "A new approach to linear filtering and prediction problems," Journal of Basic Engineering, vol. 82, no. 1, pp. 35-45, 1960.

[5] C. Z. Han, H. Y. Zhu, and Z. S. Duan, Multi-Source Information Fusion, Tinghua University Press, Beijing, China, 2nd edition, 2010.

[6] D. H. Zhou, Y. G. Xi, and Z. J. Zhang, "A suboptimal multiple fading extended Kalman filter," Acta Automatica Sinica, vol. 17, no. 6, pp. 689-695, 1991.

[7] D.-J. Jwo and S.-Y. Lai, "Navigation integration using the fuzzy strong tracking unscented Kalman filter," Journal of Navigation, vol. 62, no. 2, pp. 303-322, 2009.

[8] D.-J. Jwo and S.-H. Wang, "Adaptive fuzzy strong tracking extended Kalman filtering for GPS navigation," IEEE Sensors Journal, vol. 7, no. 5, pp. 778-789, 2007.

[9] Z. S. Duan and C. Z. Han, "A strong tracking adaptive state estimator and simulation," Journal of System Simulation, vol. 16, no. 5, pp. 1020-1023, 2004.

[10] X. He, Z. D. Wang, X. F. Wang, and D. H. Zhou, "Networked strong tracking filtering with multiple packet dropouts: algorithms and applications," IEEE Transactions on Industrial Electronics, vol. 61, no. 3, pp. 1454-1463, 2014.

[11] Y. Liang, Q. Pan, Y.-G. Jia, and D.-H. Zhou, "Strong-tracking multiple model estimator," Acta Electronica Sinica, vol. 30, no. 1, pp. 34-37, 2002.

[12] M. Boutayeb and D. Aubry, "A strong tracking extended Kalman observer for nonlinear discrete-time systems," IEEE Transactions on Automatic Control, vol. 44, no. 8, pp. 1550-1556, 1999.

[13] D.-J. Jwo and C.-M. Huang, "An adaptive fuzzy strong tracking Kalman filter for GPS/INS navigation," in Proceedings of the 33rd Annual Conference of the IEEE Industrial Electronics Society (IECON '07), pp. 2266-2271, IEEE, November 2007.

[14] M. L. Bai, D. H. Zhou, and H. Schwarz, "Identification of generalized friction for an experimental planar two-link flexible manipulator using strong tracking filter," IEEE Transactions on Robotics and Automation, vol. 15, no. 2, pp. 362-369, 1999.

[15] Q.-B. Ge, W.-B. Li, and C.-L. Wen, "SCKF-STF-CN: a universal nonlinear filter for maneuver target tracking," Journal of Zhejiang University: Science C, vol. 12, no. 8, pp. 678-686, 2011.

[16] D.-J. Jwo, C.-F. Yang, C.-H. Chuang, and T.-Y. Lee, "Performance enhancement for ultra-tight GPS/INS integration using a fuzzy adaptive strong tracking unscented Kalman filter," Nonlinear Dynamics, vol. 73, no. 1-2, pp. 377-395, 2013. 


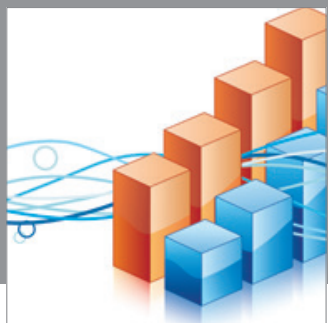

Advances in

Operations Research

mansans

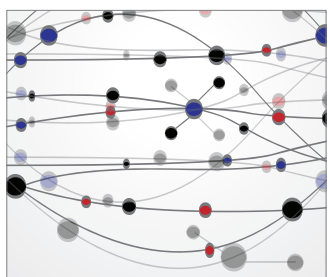

The Scientific World Journal
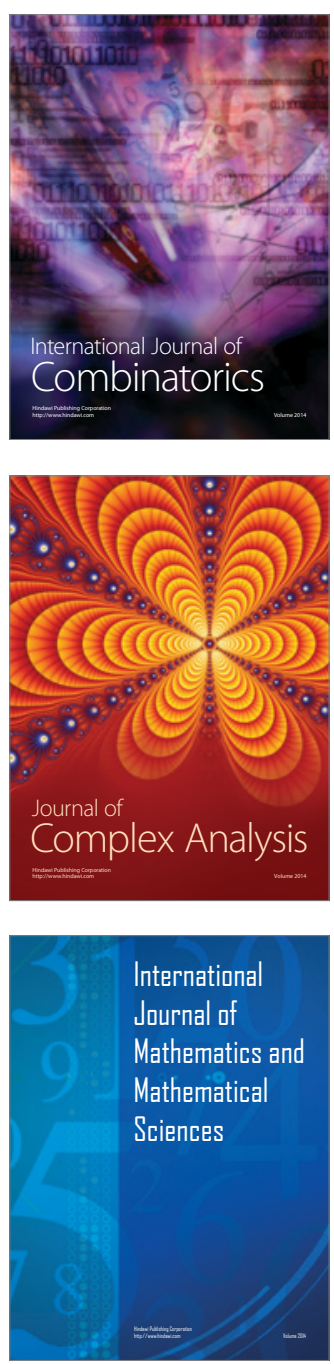
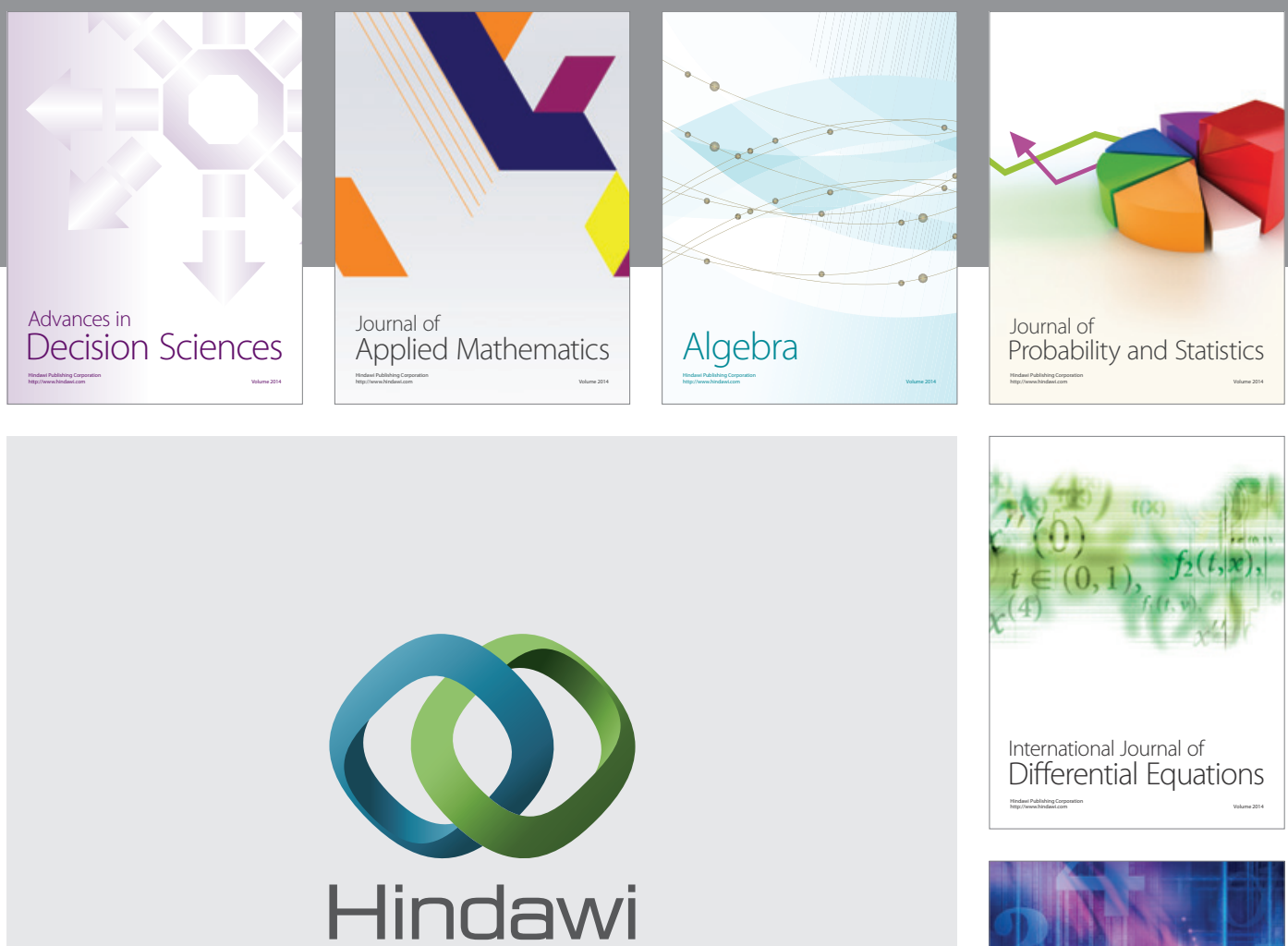

Submit your manuscripts at http://www.hindawi.com
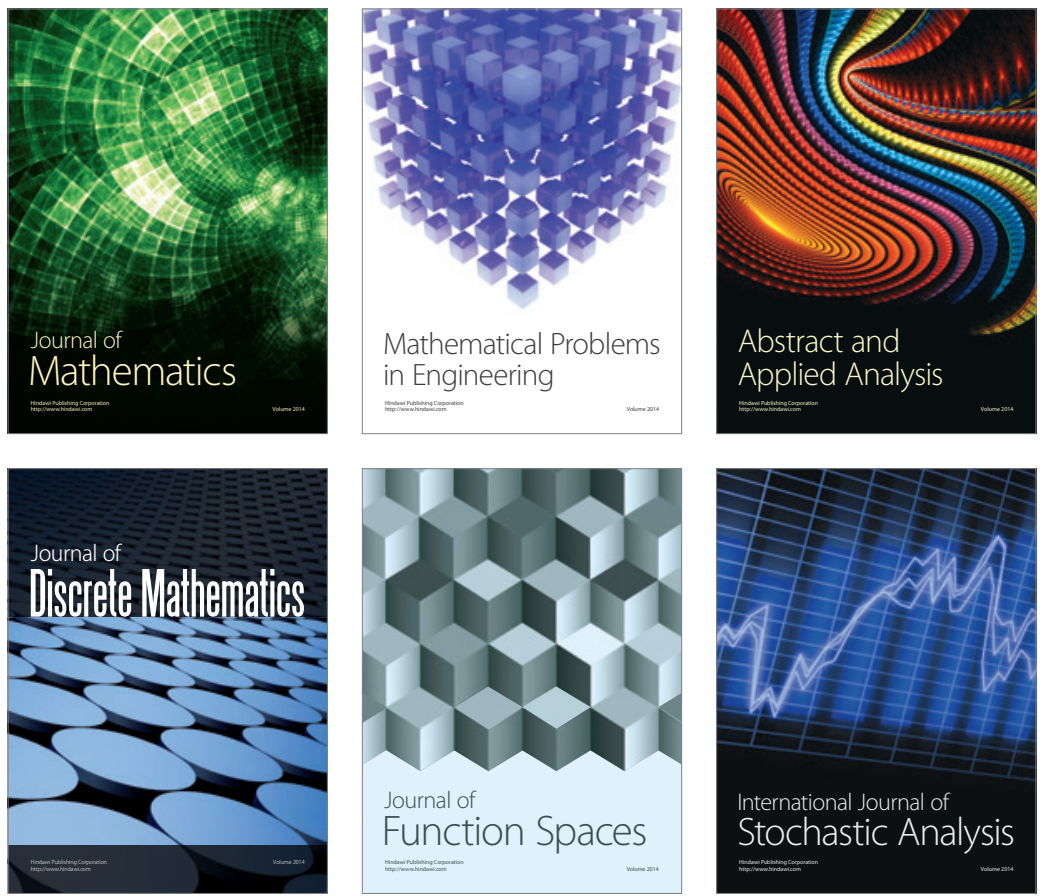

Journal of

Function Spaces

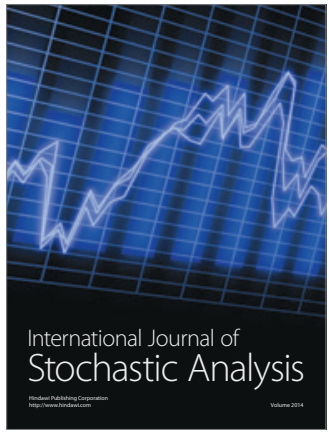

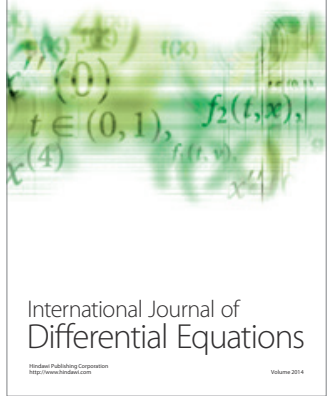
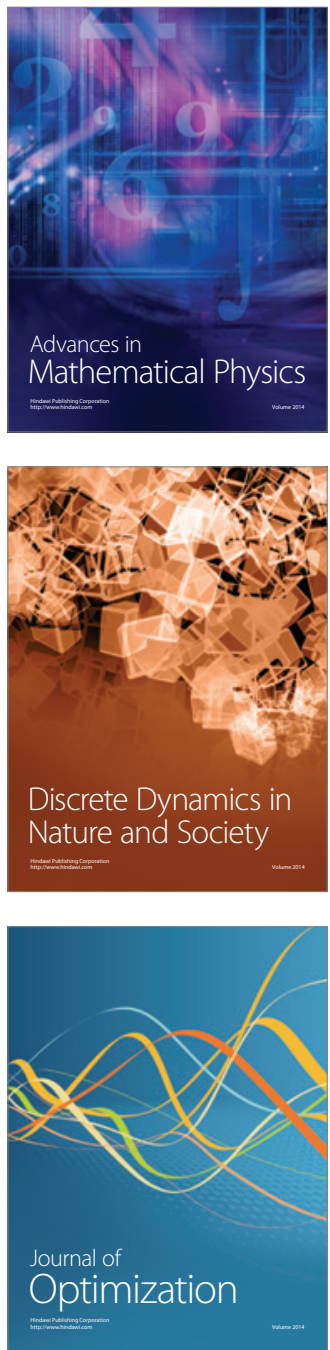